\title{
Editorial
}

\section{Vorwort der Herausgeber}

Die jährlich stattfindenden Treffen der American Society of Clinical Oncology bieten das weltweit größte Forum zur Diskussion neuester Forschungsgebiete und zur Weiterbildung in der klinischen Onkologie. Im Jahr 1999 nahmen mehr als 20,000 Onkologen am Treffen in Atlanta, Georgia, teil. Um die dort vorgestellten Ergebnisse einem breiten Fachkollegenkreis in Deutschland zugänglich zu machen, wurde gemeinsam mit der Firma Rhone-Poulenc Rorer Arzneimittel GmbH eine wissenschaftliche Tagung der Universitäts-Frauenkliniken Kiel und Hamburg sowie der Frauenklinik des Henrietten-Stiftes in Hannover durchgeführt. Namhafte Onkologen berichteten über die neuesten Entwicklungen auf dem Gebiet der gynäkologischen Onkologie.

Der Erkenntnisgewinn beim Mammakarzinom, über den dieses Jahr in Atlanta berichtet wurde, stand dabei im Vordergrund. Neben den neuesten Ergebnissen zur postoperativen Strahlentherapie, zur primär systemischen Therapie, zur adjuvanten medikamentösen Ovarektomie und zu neuesten Ansätzen bei der Behandlung des metastasierten Mammakarzinoms wurden auch die Daten zur Kontroverse bei der axillären Lymphonodektomie und zur Behandlung des duktalen Carcinoma in situ diskutiert. Großen Raum nahm auf dem ASCO-Kongreß die Diskussion der Ergebnisse der Hochdosis-Chemotherapie ein. Dabei stand außer Frage, daß die laufende «German Breast
Cancer Dose Intensity Study» (GEBDIS) fortgesetzt werden müsse. Die Behandlung des Zervixkarzinoms kam ebenso zur Sprache wie die medikamentöse Therapie des Ovarialkarzinoms. So wurde über das Screening und die Therapie des frühen Zervixkarzinoms sowie über den Einsatz der Taxane beim Ovarialkarzinom und über die entsprechenden Studienergebnisse referiert; hier wurden insbesondere auch die Resultate der AGO-Studien erwähnt. Abschließend wurden gentherapeutische Ansätze und damit die modernsten Therapiestrategien in der gynäkologischen Onkologie vorgestellt.

Im folgenden werden die Referate zusammenfassend dargestellt. Der Versuch einer umspannenden Wertung muß scheitern, da das Ergebnisspektrum außerordentlich umfangreich ist. Feststellen können wir aber, daß die Fortschritte in der Therapie gynäkologischer Malignome zwar langsam, aber doch stetig sind. Eine sachgemäße operative und medikamentöse Therapie gründet sich heute auf eine durch wissenschaftliche Studien breit gelegte Basis. Die Einführung neuer Medikamente, die in zahlreichen Studien überprüft wurden - und hierzu zählt zweifelsohne Docetaxel - haben für die Patientinnen zu einer verbesserten Lebenssituation geführt.

Walter Jonat, Kiel Fritz Jänicke, Hamburg Jörn Hilfrich, Hannover

\begin{tabular}{ll}
\hline KARGER & @ 1999 S. Karger GmbH, Freiburg \\
Fax +49 7614520714 & Accessible online at: \\
$\begin{array}{l}\text { E-mail kargergmbh@aol.com } \\
\text { www.karger.com }\end{array}$ & http://BioMedNet.com/karger
\end{tabular}

